\title{
Produção e qualidade de frutos de tomateiro cultivado em substrato com zeólita
}

\author{
Alberto C. de Campos Bernardii'; Carlos Guarino Werneck²; Patrick Gesualdi Haim²; Neide Botrel³; João \\ Oiano-Neto ${ }^{4}$; Marisa Bezerra de Mello Monte ${ }^{5}$; Maria Regina Verruma-Bernardi ${ }^{6}$ \\ ${ }^{1}$ Embrapa Pecuária Sudeste, C. Postal 339, 13560-970 São Carlos-SP; ${ }^{2}$ Alunos pós-graduandos, UFRRJ, Seropédica-RJ; ${ }^{3}$ Embrapa \\ Hortaliças, C. Postal 218, 70359-970 Brasília-DF; ${ }^{4}$ Embrapa Agroindústria de Alimentos, Rio de Janeiro-RJ; ${ }^{5}$ Centro de Tecnologias \\ Minerais, Rio de Janeiro-RJ; 6Depto. Tecnologia Agroindustrial e Sócio-EconômicoRural, Araras-SP; alberto@cppse.embrapa.br
}

\section{RESUMO}

Avaliou-se a produção e a qualidade dos frutos de tomateiro cv. Finestra, cultivado em substrato com zeólita enriquecida com N, P e K. Os tratamentos utilizados foram quatro doses $(20 ; 40 ; 80$ e $160 \mathrm{~g}$ por vaso) de zeólitas enriquecidas com $\mathrm{H}_{3} \mathrm{PO}_{4}$ /apatita, $\mathrm{KNO}_{3}$ e $\mathrm{KH}_{2} \mathrm{PO}_{4}$, além de uma testemunha cultivada em solução nutritiva. Foram avaliados a produção de frutos por vaso, firmeza, sólidos totais, $\mathrm{pH}$, acidez titulável e ácido ascórbico dos frutos, dos 80 aos 90 dias de cultivo. O fornecimento de nutrientes através do mineral zeólita enriquecido com $\mathrm{N}, \mathrm{P}$ e K comprovou ser uma alternativa para o aumento da produção. As maiores produções foram obtidas nos tratamentos com adição de $\mathrm{P}$ e $\mathrm{K}$ e nas maiores doses de zeólita ( 160 e $80 \mathrm{~g}$ por vaso). A produção de frutos foi 11 a $17 \%$ maior em relação à testemunha cultivada com solução nutritiva. Houve efeitos positivos das zeólitas enriquecidas com fontes de fósforo sobre a firmeza e efeito negativo sobre o $\mathrm{pH}$. A firmeza dos frutos variou $104 \%$ entre tratamentos, de 7,06 N (ZNK 160) a 14,38 (ZPK 40). O aumento da disponibilidade de potássio contribuiu para o aumento do teor de ácido ascórbico dos frutos.

Palavras-chave: Lycopersicon esculentum, estilbita, acidez titulável, ácido ascórbico, firmeza, sólidos solúveis totais, $\mathrm{pH}$.

\section{ABSTRACT} zeolite

Yield and fruit quality of tomato grown in substrate with

We evaluated yield and quality of tomato fruits, cv. Finestra, grown in a zeolite substrate enriched with $\mathrm{N}, \mathrm{P}$ and $\mathrm{K}$. Treatments comprised four levels $(20 ; 40 ; 80$ and $160 \mathrm{~g}$ per pot) of zeolite enriched with $\mathrm{H}_{3} \mathrm{PO}_{4}$ /apatite, $\mathrm{KNO}_{3}$ and $\mathrm{KH}_{2} \mathrm{PO}_{4}$, and a control grown in a nutrient solution. Fruit production, firmness, total soluble solids, $\mathrm{pH}$, titratable acidity and ascorbic acid were evaluated from 80 to 90 days of plant cultivation. Nutrients supplied through the mineral zeolite enriched with $\mathrm{N}, \mathrm{P}$ and $\mathrm{K}$ was an adequate alternative to increase the production. Higher fruit production was obtained with addition of $\mathrm{P}$ and $\mathrm{K}$ and higher zeolite dosis (160 and $80 \mathrm{~g}$ per pot). Fruit production was $11 \%$ and $17 \%$ higher when compared to the plants grown in nutritive solution (check treatment). Positive effects were observed in P-enriched zeolites in relation to fruit firmness, and negative effects over fruit $\mathrm{pH}$. Fruit firmness varied $104 \%$ among treatments, from 7.06N (ZNK 160) to $14.38 \mathrm{~N}$ (ZPK 40). The increase of potassium availablity increased the ascorbic acid levels of the fruits.

Keywords: Lycopersicon esculentum, stilbite, titratable acidity, ascorbic acid, firmness, total soluble solids, $\mathrm{pH}$.

\section{(Recebido para publicação em 25 de agosto de 2006; aceito em 18 de maio de 2007)}

$\mathrm{O}$ tomate é uma das mais importantes hortaliças no mundo, tanto na área cultivada como no seu valor comercial. O Brasil ocupa o oitavo lugar entre os países produtores, sendo esta hortaliça a segunda em importância econômica no país. Os frutos destinam-se especialmente para o processamento, mas também para o consumo in natura (Filgueira, 2000).

O mercado hortícola tem se tornando mais exigente, demandando alternativas viáveis para o aumento quantitativo da produção e a manutenção do fornecimento o ano todo, especialmente a obtenção de produtos de qualidade. Isso tem levado os produtores a buscar novos sistemas de cultivo, em alternativa ao sistema tradicional a campo, como os protegidos (túneis e estufas) e o hidropônico. Existe ainda a possibilida- de do cultivo zeopônico, no qual plantas são cultivadas em substrato artificial composto pelo mineral zeólita misturados a rochas fosfáticas, funcionando como um sistema de liberação controlada e renovável de nutrientes para as plantas (Allen et al., 1995; Mumpton, 1999).

As zeólitas são alumino-silicatos cristalinos hidratados de metais alcalinos ou alcalinos-terrosos, estruturados em redes cristalinas tridimensionais rígidas, formadas por tetraedros de $\mathrm{AlO}_{4}$ e $\mathrm{SiO}_{4}$. Caracterizam-se pela facilidade de reter e liberar água e trocar cátions sem modificar sua estrutura (Mumpton, 1999; Kithome et al., 1999). Esta estrutura apresenta propriedades de adsorção e capacidade de troca de íons, proporcionando o uso potencial, seja no campo ou em cultivo com substratos (Harland et al., 1999). Existem mais de 40 espécies de zeólitas naturais, sendo a clinoptilolita aparentemente a mais abundante tanto nos solos como em sedimentos (Ming \& Dixon, 1987). No Brasil os maiores reservatórios de zeólita estão no vale do Parnaíba (Rezende \& Angélica, 1991), no qual predomina a forma estilbita.

Este mineral apresenta três propriedades principais, que lhe conferem grande interesse para uso na agricultura: alta capacidade de troca de cátions, alta capacidade de retenção de água livre nos canais e alta habilidade na adsorção. Assim, a zeólita pode atuar melhorando a eficiência do uso de nutrientes através do aumento da disponibilidade de P da rocha fosfática, melhorar o aproveitamento do $\mathrm{N}\left(\mathrm{NH}_{4}^{+}\right.$e $\left.\mathrm{NO}_{3}^{-}\right)$e reduzir as perdas por lixiviação dos cátions 
trocáveis, especialmente $\mathrm{K}^{+}$e também como um fertilizante de liberação lenta (Allen et al., 1995; Notario-Del-Pino et al., 1994; Barbarick et al., 1990, Harland et al., 1999, Gül et al., 2005). De acordo com Leggo (2000), em função da afinidade da zeólita por nutrientes, este mineral pode ser utilizado em substratos para estimular o crescimento das plantas. A mistura de zeólitas com fertilizantes também apresentou efeitos positivos sobre o crescimento de plantas de alface (Gül et al., 2005) e tomate (Valente et al., 1986).

As características de qualidade do tomate dependem da cultivar, condições de cultivo, ponto de maturação na colheita, condições de armazenagem, transporte e embalagem. O tomateiro apresenta boa resposta à adubação (Filgueira, 2000); por isso, entre os vários fatores que podem limitar a produtividade e diminuir a qualidade dos frutos, as deficiências nutricionais estão entre as principais. Para obter-se altas produtividades e frutos com qualidade é necessário o fornecimento balanceado de nutrientes. Entre os indicadores que servem como parâmetro de qualidade do fruto, pode-se citar a cor, aparência, firmeza, peso, sólidos solúveis totais, $\mathrm{pH}$ e acidez titulável (Gayet et al., 1995).

O objetivo deste trabalho foi avaliar a produção e a qualidade dos frutos de tomateiro cultivado em substrato com zeólita enriquecida com N, P e K.

\section{MATERIAL E MÉTODOS}

O experimento foi conduzido em casa-de-vegetação da Embrapa Solos no Rio de Janeiro, em vasos com $3 \mathrm{~kg}$ com substrato inerte composto de areia lavada com água destilada e ácido clorídrico diluído $(3: 1 \mathrm{v} / \mathrm{v})$.

A zeólita foi coletada na Bacia do Parnaíba, no Maranhão, a qual representa o principal depósito de zeólita natural do País, com potencial de aproveitamento econômico (Rezende \& Angélica, 1991). O material coletado foi concentrado em mesa vibratória, resultando em um produto com $84 \%$ de zeólita estilbita e com capacidade de troca de cátions de 2,5 $\mathrm{cmol}_{\mathrm{c}} \mathrm{g}^{-1}$. A fórmula química determinada da zeólita foi: $(\mathrm{CaO})_{0,82}\left(\mathrm{Na}_{2} \mathrm{O}\right)_{0,19}\left(\mathrm{~K}_{2} \mathrm{O}\right)_{0,15}(\mathrm{MgO})_{0,56}$ $\left(\mathrm{Fe}_{2} \mathrm{O}_{3}\right)_{0,30}\left(\mathrm{TiO}_{2}\right)_{0,11}\left(\mathrm{Al}_{2} \mathrm{O}_{3}\right)_{1,85}\left(\mathrm{SiO}_{2}\right)_{16}$ $\left(\mathrm{H}_{2} \mathrm{O}\right)_{4,7}$. A zeólita concentrada foi enriquecida através da incubação com soluções contendo $\mathrm{H}_{3} \mathrm{PO}_{4} 1,0 \mathrm{~mol} \mathrm{~L}-1$ $(\mathrm{ZP}), \mathrm{KNO}_{3} 0,5 \mathrm{~mol} \mathrm{~L}^{-1}(\mathrm{ZNK}) \mathrm{e}$ $\mathrm{K}_{2} \mathrm{HPO}_{4} 1,0 \mathrm{~mol} \mathrm{~L}-1$ (ZPK). A relação utilizada foi de 1:40 por 24 horas, com temperatura e agitação constantes. Após a incubação, a suspensão foi filtrada e o material sólido desidratado a $100^{\circ} \mathrm{C}$. A zeólita enriquecida com $\mathrm{H}_{3} \mathrm{PO}_{4}$ recebeu, ainda, a adição de fosfato natural (34\% de $\left.\mathrm{P}_{2} \mathrm{O}_{5}\right)$ na proporção de $2: 1(\mathrm{~m} / \mathrm{m})$.

O delineamento experimental foi em blocos ao acaso, com três repetições. Os vasos foram cultivados com o tomate (Lycopersicon esculentum, Mill ) cv. Finestra. Avaliaram-se os doze tratamentos com melhor aspecto dos frutos: Z20, Z40, ZNK160, ZPK20, ZPK40, ZPK80, ZPK160, ZP20, ZP40, ZP80, ZP160, e a testemunha (sem adição de zeólita). As doses e concentração de nutrientes em cada um deles encontramse descritos na Tabela 1. A zeólita apresentava traços de outros elementos, entre eles o fósforo, por isso o material concentrado apresentou uma pequena porcentagem de $\mathrm{P}$ disponível. A testemunha recebeu todos os nutrientes necessários para o desenvolvimento das plantas através de uma solução nutritiva, com a composição $\left(\mathrm{mg} \mathrm{L}^{-1}\right)$ : $\mathrm{N}=210$; $\mathrm{P}=31 ; \mathrm{K}=234 ; \mathrm{Ca}=200 ; \mathrm{Mg}=48 ; \mathrm{S}$ $=65 ; \mathrm{B}=0,5 ; \mathrm{Cu}=0,02 ; \mathrm{Fe}=5,0 ; \mathrm{Mn}$ $=0,5 ; \mathrm{Zn}=0,2$, e Mo $=0,02$. Essa solução foi preparada segundo Sarruge (1975). O fornecimento foi realizado manualmente, em quarenta aplicações de $100 \mathrm{~mL}$ de solução nutritiva por vaso com duas plantas, totalizando 4,0 L por vaso. As aplicações ocorreram ao longo de todo o ciclo de cultivo, sendo que nos primeiros 30 dias de cultivo o intervalo de fornecimento foi de três dias; nos 60 dias seguintes o intervalo foi de 1 dia. Nos dias em que não se forneceu solução, as plantas receberam água da irrigação. Quando a zeólita utilizada não possuía um dos macronutrientes em teste $(\mathrm{N}, \mathrm{P}$ ou $\mathrm{K})$ este era adicionado na forma de solução, na mesma concentra- ção fornecida à testemunha. Desse modo todos os tratamentos receberam as mesmas quantidades de $\mathrm{Ca}, \mathrm{Mg}, \mathrm{S}, \mathrm{B}, \mathrm{Cu}$, $\mathrm{Fe}, \mathrm{Mn}$, Mo e Zn.

Os tratamentos foram iniciados em agosto de 2003 com o transplante, para os vasos de cultivo, de duas mudas do tomateiro aos 30 dias após a germinação. Os frutos foram coletados para análise quando atingiram a completa coloração vermelha. A colheita iniciou-se aos 80 dias após transplante (DAT) e terminou aos 90 DAT, sendo que todos os frutos foram pesados. Nos frutos maduros colhidos foram avaliados os sólidos solúveis totais e $\mathrm{pH}$ (Instituto Adolfo Lutz, 1985), acidez total titulável (International Organization of Standardization - ISO, 1998), concentração de ácido ascóbico (Ashoor et al., 1984), e a firmeza (com o uso de um penetrômetro McCormick, modelo FT 327 , com ponteiras de $8 \mathrm{~mm}$ ). Os resultados de produção e de qualidade de frutos foram analisados estatisticamente através da análise de variância e teste de Duncan (5\%).

\section{RESULTADOS E DISCUSSÃO}

As maiores produções de frutos foram obtidas nos tratamentos com adição de P e K (ZP e ZPK) e nas maiores doses de zeólita (160 e 80 g por vaso), as quais foram significativamente 11 a $17 \%$ maiores que a testemunha, que recebeu todos os nutrientes na forma de solução nutritiva (Tabela 2). Nanadal et al. (1998) também verificaram aumentos da produção do tomateiro com o fornecimento dessas concentrações destes dois macronutrientes. Estas porcentagens de aumento são concordantes com os obtidos por Leggo (2000), que comparou plantas cultivadas em substratos com e sem zeólita, e verificou aumentos de $19 \%$ na produção de matéria seca na presença do mineral. Nesse trabalho verificou-se as maiores produtividades com a aplicação das maiores doses de potássio, devido à influência deste elemento no tamanho dos frutos, uma vez que sua deficiência acarreta a produção de frutos menores e menor teor de licopeno (Knee, 2002). Estes resultados 
Tabela 1. Nutrientes adicionados à zeólita, dose de zeólita por vaso e quantidade de nutrientes fornecidas nos tratamentos utilizados (Nutrient-enriched zeolite, zeolite dosis per pot and quantity of nutrients in the treatments). Rio de Janeiro, Embrapa/Centro de tecnologias minerais, 2003.

\begin{tabular}{|c|c|c|c|c|c|c|c|c|}
\hline \multirow[t]{2}{*}{ Tratamento } & \multirow[t]{2}{*}{ Enriquecimento } & \multicolumn{3}{|c|}{ Concentração* $\left(\mathrm{mg} \mathrm{kg}^{-1}\right)$} & \multirow{2}{*}{$\begin{array}{c}\text { Zeólita } \\
\text { (g por vaso) }\end{array}$} & \multicolumn{3}{|c|}{$\begin{array}{l}\text { Quantidade fornecida pela zeólita (mg } \\
\text { por vaso) }\end{array}$} \\
\hline & & $\mathrm{N}\left(\mathrm{N}-\mathrm{NO}_{3}\right)$ & $\mathbf{P}$ & $\mathbf{K}$ & & $\mathrm{N}\left(\mathrm{N}-\mathrm{NO}_{3}\right)$ & $\mathrm{P}_{2} \mathrm{O}_{5}$ & $\mathrm{~K}_{2} \mathrm{O}$ \\
\hline Z 20 & zeólita concentrada & 0,1 & 17 & 3,9 & 20 & 0,002 & 0,8 & 0,1 \\
\hline Z 40 & zeólita concentrada & 0,1 & 17 & 3,9 & 40 & 0,004 & 1,6 & 0,2 \\
\hline ZP 20 & zeólita $+\mathrm{H}_{3} \mathrm{PO}_{4}+$ apatita & 0,2 & 7130 & 3,9 & 20 & 0,004 & 327 & 0,1 \\
\hline ZP 40 & zeólita $+\mathrm{H}_{3} \mathrm{PO}_{4}+$ apatita & 0,2 & 7130 & 3,9 & 40 & 0,008 & 653 & 0,2 \\
\hline ZP 80 & zeólita $+\mathrm{H}_{3} \mathrm{PO}_{4}+$ apatita & 0,2 & 7130 & 3,9 & 80 & 0,016 & 1306 & 0,4 \\
\hline ZP 160 & zeólita $+\mathrm{H}_{3} \mathrm{PO}_{4}+$ apatita & 0,2 & 7130 & 3,9 & 160 & 0,032 & 2612 & 0,8 \\
\hline ZPK 20 & zeólita $+\mathrm{KH}_{2} \mathrm{PO}_{4}$ & 0,1 & 11289 & 41925 & 20 & 0,002 & 517 & 1010 \\
\hline ZPK 40 & zeólita $+\mathrm{KH}_{2} \mathrm{PO}_{4}$ & 0,1 & 11289 & 41925 & 40 & 0,004 & 1034 & 2021 \\
\hline ZPK 80 & Zeólita $+\mathrm{KH}_{2} \mathrm{PO}_{4}$ & 0,1 & 11289 & 41925 & 80 & 0,008 & 2068 & 4042 \\
\hline ZPK 160 & Zeólita $+\mathrm{KH}_{2} \mathrm{PO}_{4}$ & 0,1 & 11289 & 41925 & 160 & 0,016 & 4136 & 8084 \\
\hline ZNK 160 & Zeólita + $\mathrm{KNO}_{3}$ & 93796 & 17 & 15210 & 160 & 15008 & 6,2 & 2932 \\
\hline
\end{tabular}

*Extraídos em pasta de saturação (Embrapa, 1997).

Tabela 2. Produção e parâmetros de qualidade de frutos do tomateiro cv. Finestra cultivado em substrato com zeólita. Média de três repetições (Yield and quality parameter of tomato fruits, cv. Finestra, cultivated in a zeolite substrate. Average value of three replications). Rio de Janeiro, Embrapa/Centro de tecnologias minerais, 2003.

\begin{tabular}{|c|c|c|c|c|c|c|}
\hline Tratamentos & $\begin{array}{l}\text { Produção } \\
\text { (g por vaso) }\end{array}$ & $\begin{array}{c}\text { Firmeza } \\
(\mathrm{N})\end{array}$ & $\begin{array}{c}\text { Sólido totais } \\
\text { ( }{ }^{\circ} \text { Brix) }\end{array}$ & $\mathrm{pH}$ & $\begin{array}{l}\text { Acidez titulável } \\
(\% \text { ac. Cítrico) }\end{array}$ & $\begin{array}{l}\text { Ácido ascórbico(1) } \\
\left(\mathrm{mg} \mathrm{100}^{-1}\right)\end{array}$ \\
\hline Z 20 & $589,0 \mathrm{cde}$ & 7,38 ef & 3,50 & $4,21 \mathrm{abc}$ & $0,60 a$ & $16,54 \mathrm{~b}$ \\
\hline Z 40 & $587,6 \mathrm{cde}$ & $7,08 \quad f$ & 3,60 & $4,14 \mathrm{bcd}$ & $0,53 a b$ & $17,68 \mathrm{~b}$ \\
\hline ZP 20 & $446,3 \quad f$ & 8,17 def & 3,63 & $4,07 \mathrm{~cd}$ & $0,40 a b$ & $16,65 b$ \\
\hline ZP 40 & $300,1 \quad \mathrm{~g}$ & 9,56 cdef & 3,40 & $4,10 \mathrm{~cd}$ & $0,40 a b$ & $16,83 \mathrm{~b}$ \\
\hline ZP 80 & 521,3 def & $12,20 a b c$ & 3,53 & $4,12 \mathrm{~cd}$ & $0,33 \mathrm{~b}$ & $16,59 \mathrm{~b}$ \\
\hline ZP 160 & $711,2 a b$ & $12,95 a b$ & 3,35 & $4,17 \mathrm{abcd}$ & $0,33 \mathrm{~b}$ & $19,12 b$ \\
\hline ZPK 20 & $339,6 \quad \mathrm{~g}$ & 10,46 bcde & 3,20 & $4,28 a b$ & $0,43 a b$ & $7,84 \mathrm{c}$ \\
\hline ZPK 40 & 479,0 ef & $14,38 a$ & 3,47 & $4,32 a$ & $0,40 a b$ & $10,26 \mathrm{c}$ \\
\hline ZPK 80 & $616,1 \mathrm{bcd}$ & $11,13 \mathrm{bcd}$ & 3,60 & $4,18 \mathrm{abcd}$ & $0,37 a b$ & $19,22 \mathrm{~b}$ \\
\hline ZPK 160 & $751,9 a$ & 7,74 ef & 3,77 & $4,05 \quad d$ & $0,44 a b$ & $24,13 a$ \\
\hline ZNK 160 & $114,7 \quad h$ & $7,06 \quad f$ & 3,40 & $4,18 a b c d$ & $0,51 a b$ & $15,27 \mathrm{~b}$ \\
\hline Testemunha & $641,3 \mathrm{bc}$ & 7,36 ef & 3,67 & $4,13 \mathrm{bcd}$ & $0,59 a b$ & $16,81 \mathrm{~b}$ \\
\hline Teste F & $24,31^{* * *}$ & $4,62^{* * *}$ & $0,68^{\text {N.S. }}$ & $3,46^{* *}$ & $2,45^{*}$ & $3,38^{* *}$ \\
\hline CV\%(2) & 17,00 & 23,21 & 8,82 & 1,99 & 30,16 & 20,44 \\
\hline
\end{tabular}

Médias seguidas de letras diferentes diferem significativamente pelo teste de Duncan a 5\%; ${ }^{(1)}$ Concentração de ácido ascórbico com base na

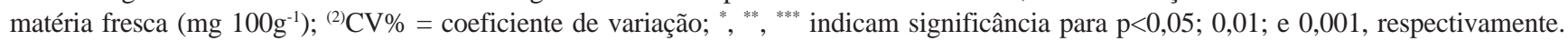
N.S. = não significativo.

demonstram o potencial para utilização do mineral zeólita enriquecido, como uma fonte de nutrientes para esta solanácea. Desta forma, há concordância com os resultados já obtidos por Notario-Del-Pino et al. (1994), Williams \& Nelson (1997) e Gül et al. (2005) para alfafa, crisântemo e alface, respectivamente, e Valente et al. (1986) para tomateiro.

A qualidade dos frutos avaliada em função da acidez titulável, $\mathrm{pH}$, firmeza e concentração de ácido ascórbico (vitamina C), foi influenciada significativamente pelos tratamentos com zeólita enriquecida. Não houve efeito dos tratamentos sobre os teores de sólidos solúveis (Tabela 2).

A ausência de diferenciação dos resultados obtida para o teor de sólidos solúveis ( ${ }^{\circ}$ Brix) concorda com os resultados de outros autores (Gul \& Sevgican, 1992; Alan et al., 1994), os quais também não verificaram diferen- ça significativa para esta característica em tomate produzido em diversos substratos e no solo. No entanto, a faixa de valores observada para os sólidos solúveis entre 3,2 e $3,77 \%$ está abaixo da faixa normalmente observada, entre 4 e 5\% (Jones, 1998; Fontes et al., 2000; Sampaio \& Fontes, 2000). De acordo com Knee (2000), os teores de sólidos solúveis aumentam com o aumento das doses de nitrogênio.

Houve diferenças significativas entre a acidez titulável, representada pela 
concentração de ácido cítrico (Tabela 2), do tratamento Z20 $(0,60 \%)$ e dos tratamentos ZP80 e ZP160 (0,33\%). Os demais tratamentos não diferiram entre si. Estes resultados indicam efeito positivo da fonte de fósforo presente nestes tratamentos (ZP80 e ZP 160) na redução destes valores, como observados por Sobulo \& Olorunda (1977). A acidez devida a ácidos orgânicos é uma característica importante que influencia a palatabilidade aos frutos, e diminui com a maturação em decorrência do processo respiratório e da conversão em açúcares (Kader et al., 1978). O aumento no fornecimento de $\mathrm{P}$ pode aumentar a diponibilidade de compostos bioquímicos que utilizam o fosfato como armazenamento de energia. Como vários processos metabólicos vitais dependem desse suprimento de energia, a nutrição adequada de fósforo tende a favorecê-los (Marschner, 1995) e desta forma incrementar a neutralização os ácidos e sintese de açúcares, tornando os frutos menos ácidos. Alan et al. (1994) também obtiveram diferenças significativas nos teores de ácido cítrico de tomate produzido em diferentes substratos. No entanto, Lacatus et al. (1995) não encontraram diferenças significativas para acidez titulável em tomate produzido em diversos substratos e no solo. Os valores médios observados para acidez titulável $(0,44 \%)$ estão acima do valor de $0,32 \%$, considerado mínimo para tomate de alta qualidade (Kader et al., 1978; Jones, 1998).

Houve diferença estatística significativa entre os tratamentos para os valores de $\mathrm{pH}$. A faixa dos valores foi de 4,07 a 4,32, com valor médio de 4,16. Apesar destas diferenças entre os tratamentos, a amplitude de variação está dentro da faixa de $\mathrm{pH}$ de 4,0 a 4,5, considerada ideal para tomates de qualidade, segundo Sapers et al. (1978) e Jones (1998). Gul \& Sevgican (1992) e Fontes et al. (2004) também não encontraram diferenças significativas no valor de $\mathrm{pH}$ do tomate produzido em casa de vegetação, em diferentes substratos e no solo. Os valores de $\mathrm{pH}$ mais baixos podem ser atribuídos à maior concentração de ácido cítrico nos frutos, uma vez

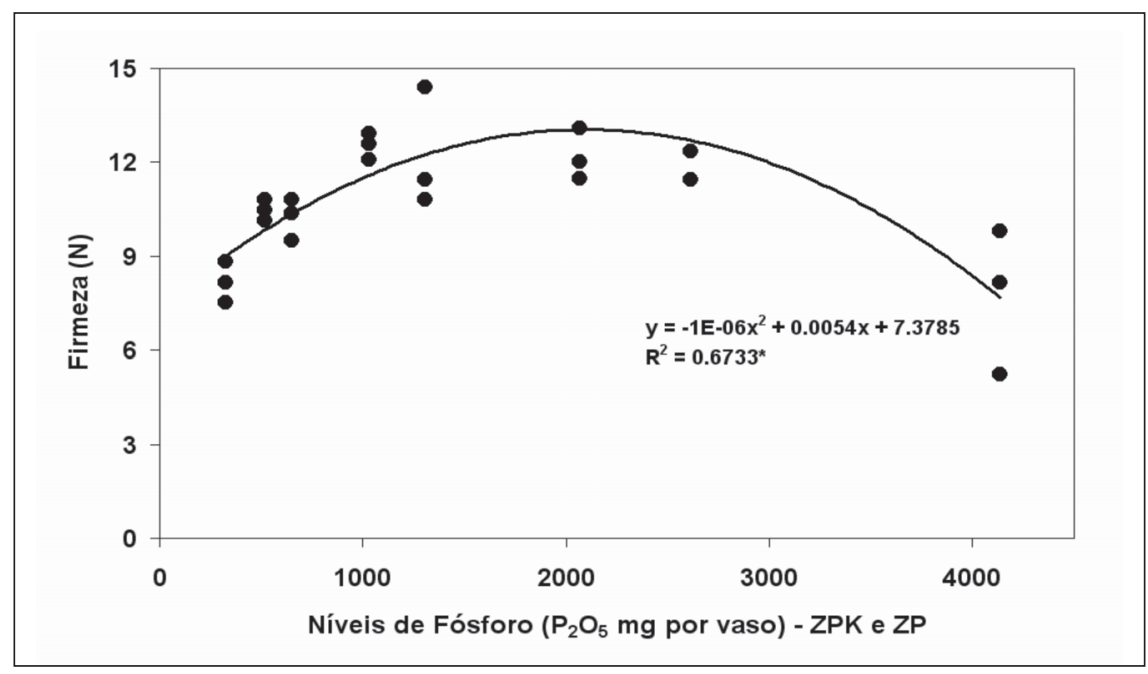

Figura 1. Valores de firmeza dos frutos do tomateiro cv. Finestra em função dos níveis de fósforo no substrato com zeólitas ZPK e ZP (Tomato fruit firmness values from cv. Finestra, as a function of levels of phosporus in the substrate, with zeolites ZPK and ZP). Rio de Janeiro, Embrapa/Centro de tecnologias minerais, 2003.

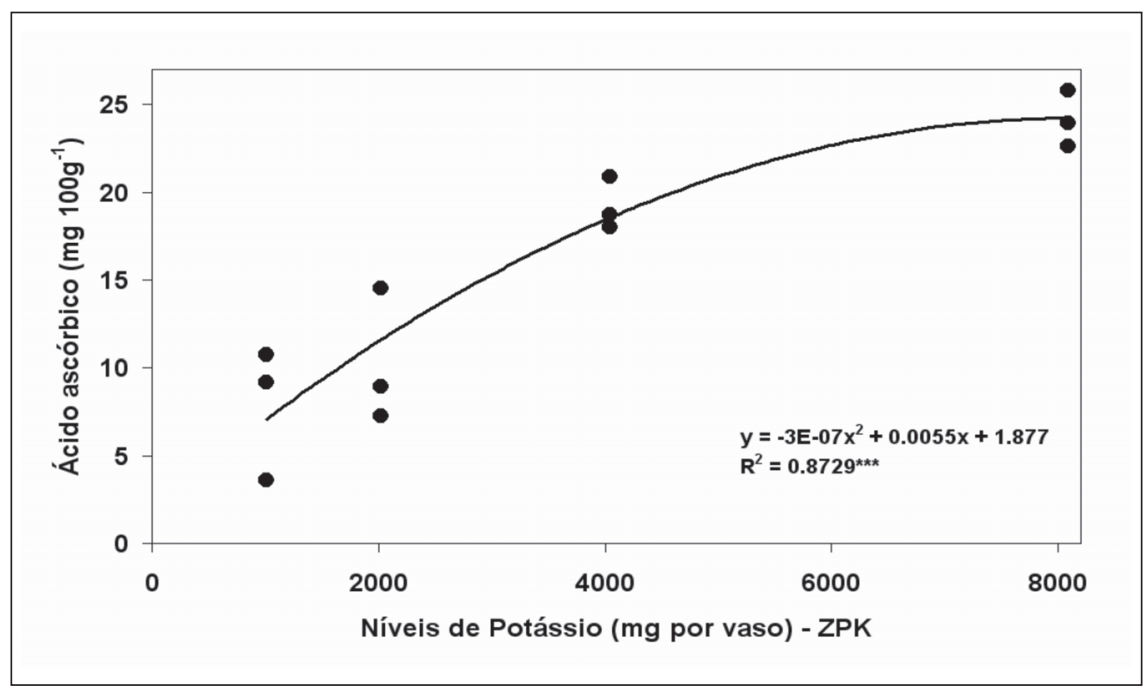

Figura 2. Concentração de ácido ascórbico nos frutos do tomateiro cv. Finestra em função dos níveis de potássio no substrato com zeólitas ZPK (Concentration of ascorbic acid in tomato fruits, cv. Finestra, as a result of kalium levels in the substrate with the zeolite ZPK). Rio de Janeiro, Embrapa/Centro de tecnologias minerais, 2003.

que se observou uma relação significativa $(\mathrm{p}<0,05)$ inversa com coeficiente de correlação (r) igual a 0,792 entre estas duas variáveis.

A firmeza é um dos mais importantes atributos da qualidade de frutos de tomate para consumo in natura e para o cultivo industrial. De acordo com Ahrens et al. (1990), esta é uma característica de conservação pós-colheita essencial durante o transporte e comercialização dos frutos, também re- lacionada com a capacidade de armazenamento ou "vida de prateleira". Os valores de firmeza variaram de 7,06 $\mathrm{N}$ (ZNK 160) a 14,38 N (ZPK 40), representando uma amplitude de variação de $104 \%$ (Tabela 2). Os frutos mais firmes ocorreram nos tratamentos em que a disponibilidade de fósforo era maior ZPK 40, ZP 160, ZP 80, ZPK 80 e ZPK20. Estes valores representaram aumentos significativos em relação à testemunha entre 95 e $42 \%$. Consideran- 
do-se apenas os tratamentos nos quais havia variação dos teores de $\mathrm{P}$ (zeólitas ZP e ZPK), observou-se uma tendência de resposta quadrática entre os resultados de firmeza dos frutos em função das doses de $\mathrm{P}$ fornecidas com as zeólitas (Figura 1). Sendo que o maior valor (14,2 N) foi obtido próximo da dose de $2000 \mathrm{mg}$ por vaso de $\mathrm{P}_{2} \mathrm{O}_{5}$.

As concentrações de ácido ascórbico nos frutos in natura foram afetadas significativamente pelos tratamentos (Tabela 2). Os valores obtidos estão de acordo com os descritos na literatura, nos quais as concentrações de ácido ascórbico em tomate cultivado em condições de casa-de-vegetação, variaram de 7 a $23 \mathrm{mg} 100 \mathrm{~g}^{-1}$ de matéria fresca (Dumas et al., 2003) e de 17 a $22 \mathrm{mg}$ $100 \mathrm{~g}^{-1}$ de matéria fresca em diferentes cultivares em condições de campo (Abushita et al., 2000).

Os resultados obtidos neste trabalho indicam que o fornecimento de $\mathrm{K}$ tem efeito positivo sobre a concentração de ácido ascórbico, devido ao aumento da disponibilidade do nutriente. Estas observações são concordantes com as de Solubo \& Olorunda (1977), Anac \& Colcoglu (1995), Nanadal et al. (1998), Sampaio \& Fontes (2000) e Balliu \& Ibro (2002), que descreveram que aumentos no fornecimento de potássio aumentavam os valores de ácido ascórbico nos frutos do tomateiro. Já Fontes et al. (2000) não observaram diferenças nos valores deste ácido em função da variação das doses de potássio.

O potássio apresenta importantes funções nas células vegetais como cofator enzimático, na síntese e estabilidade de proteínas e na síntese de carboidratos (Marschner, 1995). Como o ácido ascórbico é uma lactona de um ácido-açúcar, nos tratamentos onde houve maior disponibilidade deste nutriente, os frutos apresentaram maiores concentrações de ácido ascórbico, provavelmente devido ao aumento na síntese e transporte de açúcares fotossintetados. $\mathrm{Na}$ Figura 2 observa-se resposta quadrática dos valores de ácido em relação às doses de K aplicadas na adubação, sendo que o maior teor (26 mg 100 $\mathrm{g}^{-1}$ ) foi obtido na dose de $160 \mathrm{~g}$ por vaso de zeólita ZPK, representando cerca de $8 \mathrm{~g}$ de potássio por vaso. Sampaio \& Fontes (2000) obtiveram valores de 20 mg $100 \mathrm{~g}^{-1}$ com aplicações de $180 \mathrm{~kg} \mathrm{ha}^{-1}$ de potássio.

\section{REFERÊNCIAS}

ABUSHITAAA; DAOOD HG; BIACS PA. 2000. Change in carotenoids and antioxidant vitamins in tomato as a function of varietal and technological factors. Journal of Agricultural and Food Chemistry 48: 2075-2081.

AHRENS MJ; HUBER DJ. 1990. Physiology and firmness determination of ripening tomato fruit. Physiologia Plantarum 78: 8-14.

ALAN R; ZULKADIR A; PADEM H. 1994. The influence of growing media on growth, yield and quality of tomato grown under greenhouse conditions. Acta Horticulturae 366: 429-435.

ANAC D; COLCOGLU H. 1995. Response of some major crops to $\mathrm{K}$ fertilization. In: MENGEL K; KRAUSS A. (Eds.), $K$ Availability of Soils in West Asia and North Africa-Status and Perspectives. Bern: International Potash Institute, p. 235-247.

ASHOOR SH; MONTE WC; WELTY J. 1984. Liquid chromatographic determination of ascorbic acid in foods. Journal of the Association of Official Analytical Chemists 67: 78-80.

BALLIU A; IBRO V. 2002. Influence of different levels of potassium fertilizers on growth, yield and ascorbic acid content of tomato fruit grown in non-heated greenhouse. Acta Horticulturae 579: 385-388.

BARBARICK KA; LAI TM; EBERL DD. 1990. Exchange fertilizer (phosphate rock plus ammonium-zeolite) effects on sorghumsudangrass. Soil Science Society of America Journal 54: 911-916.

DUMAS Y; DADOMO M; LUCCA GD; GROLIER P; DI LUCCA G. 2003. Effects of environmental factors and agricultural techniques on antioxidant content of tomatoes. Journal of the Science of Food and Agriculture 83: 369-382.

EMBRAPA. 1997. Centro Nacional de Pesquisa de Solos. Manual de métodos de análise de solo. Rio de Janeiro: EMBRAPA/CNPS,. 212 p. (EMBRAPA-CNPS. Documentos, 1).

FILGUEIRA FAR. 2000. Novo Manual de Olericultura: agrotecnologia moderna na produção e comercialização de hortaliças. Viçosa: Editora UFV. 402p

FONTES PCR; LOURES JL; GALVÃO JCC; CARDOSO AA; MANTOVANI EC. 2004. Produção e qualidade do tomate produzido em substrato, no campo e em ambiente protegido. Horticultura Brasileira 22.

FONTES PCR; SAMPAIO RA; FINGER FL. 2000. Fruit size, mineral composition and quality of trickle-irrigated tomatoes as affected by potassium rates. Pesquisa Agropecuária Brasileira 35: 21-25.
GAYET JP; BLEINROTH EW; MATALLO M; GARCIA EEC; GARCIA AE; ARDITO EFG; BORDIN MR. 1995. Tomate para Exportação: Procedimentos de Colheita e Pós-colheita. Ministério da Agricultura, do Abastecimento e da Reforma Agrária, Secretaria do Desenvolvimento Rural, Programa de Apoio à Produção e Exportação de Frutas, Hortaliças, Flores e Plantas Ornamentais - Brasília: EMBRAPA-SPI. 34p. (Série Publicações Técnicas FRUPEX; 13).

GÜL A; EROGUL D; ONGUN AR. 2005. Comparison of the use of zeolite and perlite as substrate for crisp-head lettuce. Scientia Horticulturae 106: 464-471.

GUL A; SEVGICAN A. 1992. Effect of growing media on glasshouse tomato yield and quality. Acta Horticulturae 303: 145-150.

HARLAND J; LANE S; PRICE D. 1999. Further experiences with recycled zeolite as a substrate for the sweet pepper crop. Acta Horticulturae 481: 187-194.

INSTITUTO ADOLFO LUTZ. 1985. Normas analíticas do Instituto Adolfo Lutz. Métodos químicos e físicos para análises de alimentos. 3. ed. São Paulo. v. 1, 375 p.

INTERNATIONAL ORGANIZATION OF STANDARDIZATION - ISO. 1998. Fruit and vegetable products - Determination of titratable acidity. ISO 750.

JONES JR; JB. 1998. Tomato plant culture - in the field, greenhouse, and home garden. New York: CRC Press. 199 p.

KADER AA; MORRIS LL; CHEM P. 1978. Evaluation of two objective methods and a subjective rating scale for measuring tomato fruit firmness. Journal of the American Society for Horticultural Science 103: 70-73.

KITHOME M; PAUL JW; LAVKULICH LM; BOMKE AA. 1999. Effect of $\mathrm{pH}$ on ammonium adsorption by natural zeolite clinoptilolite. Communications on Soil Science and Plant Analysis 30: 1417-1430.

KNEE M. 2002. Fruit quality and its biological basis. Sheffield: Sheffield Academic Press. 279p.

LACATUS V; BOTES C; POPESCU N; VOICAN V. 1995. Chemical composition of tomato and sweet pepper fruits cultivated on active substrates. Acta Horticulturae 412: 169-173.

LEGGO PJ. 2000. An investigation of plant growth in an organo-zeolitic substrate and its ecological significance. Plant and Soil 219: 135-146.

MARSCHNER H. 1995. Mineral nutrition of higher plants. New York: Academic Press. $889 \mathrm{p}$.

MING DW; DIXON JB. 1987. Quantitative determination of clinoptilolite in soils by a cation-exchange capacity method. Clays and Clay Mineralogy 35: 463-468.

MUMPTON FA. 1999. La roca magica: Uses of natural previous zeolites in agriculture and industry. Proceedings of National Academy of Sciences of the United States of America 96: 3463-3470. 
NANADAL JK; RAMESH V; PANDEY UC. 1998. Effect of phosphorus and potassium on growth yield and quality of tomato. Journal of Potassium Research 14: 44-49.

NOTARIO-DEL-PINO JS; ARTEAGAPADRON IJ; GONZALEZ-MARTIN MM; GARCIA-HERNANDEZ JE. 1994. Response of alfalfa to a phillipsite-based slow-release fertilizer. Communications in Soil Science and Plant Analysis 25: 2231-2245.
REZENDE NGAM; ANGÉLICA RS. 1991. Sedimentary zeolites in Brazil. Mineralogica et Petrographica Acta 42: 71-82.

SAMPAIO RA; FONTES PCR. 2000. Composição química e qualidade de frutos do tomateiro em função da adubação potássica. Ciência Agrícola 5: 65-73.

SAPERS GM; PHILLIPS JG; PANASIUK O CARRÉ J; STONER AK; BARKSDALE T. 1978. Factors affecting the acidity of tomatoes. HostScience 13: 187-189.
SARRUGE JR. 1975. Soluções nutritivas. Summa Phytopatologica 1: 231-3.

SOBULO RA; OLORUNDA AO. 1977. The effects of nitrogen, phosphorus and potassium on the canning quality of tomatoes (Lycopersicon esculentum) in South-Western Nigeria. Acta Horticulturae 53: 171-180.

VALENTE S; BURRIESCI N; CAVALLARO S; GALVAGNO S; ZIPELLI C. 1986. Utilization of zeolite as soil conditioner in tomato growing. Zeolites 2: 271-274. 